\title{
Research on the Structural Rigidity Characteristics of a Reconfigurable TBM Thrust Mechanism
}

Younan $\mathrm{Xu}^{1^{*}}$, Xinjun Liu ${ }^{2^{*}}$ and Jiyu $\mathrm{Xu}^{3}$

\begin{abstract}
To improve the adaptability of TBMs in diverse geological environments, this paper proposes a reconfigurable Type-V thrust mechanism (V-TM) with rearrangeable working states, in which structural stiffness can be automatically altered during operation. Therefore, millions of configurations can be obtained, and thousands of instances of working status per configuration can be set respectively. Nonetheless, the complexity of configurations and diversity of working states contributes to further complications for the structural stiffness algorithm. This results in challenges such as difficulty calculating the payload compliance index and the environment adaptability index. To solve this problem, we use the configuration matrix to describe the relationship between propelling jacks under reconfiguration and adopt pattern vectors to describe the working state of each hydraulic cylinder. Then, both the dynamic compatible equation between propeller forces of the hydraulic cylinders and driving forces, and the kinematic harmonizing equation between the hydraulic cylinder displacements and their deformations are established. Next, we derive the stiffness analytical equation using Hooke's law and the Jacobian Matrix. The proposed approach provides an effective algorithm to support structural rigidity analysis, and lays a solid theoretical foundation for calculating the performance indexes of the V-TM. We then analyze the rigidity characteristics of typical configurations under different working states, and obtain the main factors affecting structural stiffness of the V-TM. The results show the deviation degree of structural parameters in hydraulic cylinders within the same group, and the working status of propelling jacks. Finally, our constructive conclusions contribute valuable information for matching and optimization by drawing on the factors that affect the structural rigidity of the $\mathrm{V}-\mathrm{TM}$.
\end{abstract}

Keywords: Reconfigurable TBM thrust mechanism, Structural rigidity characteristics, Configuration matrix, Pattern vector, Kinematic harmonizing equation, Dynamic compatible equation, Structural stiffness equations

\section{Introduction}

A Tunnel Boring Machine (TBM) consists of highly complex mechatronic equipment used to cut a full-face tunnel with hard rock. It bears heavy payload [1], interrupt dynamical payload [2] and intense impact payload [3] from diverse geological environments. This results in

\footnotetext{
*Correspondence: xyn@ecjtu.edu.cn; xinjunliu@tsinghua.edu.cn

1 School of Mechantronics and vehicle Engineering, East China Jiaotong

University, Nanchang 330013, China

2 Department of Mechanical Engineering, Tsinghua University,

Beijing 100084, China

Full list of author information is available at the end of the article
}

a slowdown in cutting velocity, and an expected reduction in the lifetime of key components and parts, or a deviation in the trajectory of the cutter head, especially for tunnels with hard rock [4]. To speed up TBM cutting velocity for hard rock, a stronger cutter system should be developed [5], with the layout optimized [6-8]. Furthermore, the great cutting forces and cutting torques should go through the thruster mechanism (TM). However, this can also negatively affect the key components and parts. To solve this conflict, payload compliance design theory for thrusters of shield machines is established and the design method presented by the authors [9]. Payload compliance of shield machines [10] and the compliance 
of the hydraulic system of shield machines [11] have been widely studied, though hydraulic stiffness was studied by Snowdown, et al. as early as 1983 [12].

The main functions of a TBM thruster include thrusting, gripping, and regripping. Its configuration is much more complex than that of a shield machine [4]. Thus, the question of how to design and/or develop a new kind of TBM thrust system, with high adaptability [13] in diverse geological environments, and the capability to effectively operate during different steps of the working process, has become a major problem to be solved. This paper will focus on the structural rigidity characteristics of a new type of TBM thruster to help calculate the payload compliance index of the TM.

In fact, to improve the compliance of the TM of a shield machine in diverse geological environments, several research works have been proposed. Currently, the active compliance performance of a given TBM could be improved by updating its control methods $[14,15]$, while its passive compliance performance could be improved through several different approaches. Such as, to optimize the cuts layout on the cutter head $[6,7]$, to rearrange the group dividing of hydraulic cylinders [16-18], and to optimize the layout of hydraulic cylinders [19-21].

As shown in Figure 1, to achieve this, a new V-TM equipped with 12 jacks (or hydraulic cylinders) is proposed $[4,16,22]$. Based on the reconfiguration method, this V-TM consists of millions of configurations, each configuration operates in thousands of working states, and its structural stiffness can be altered automatically during operation.

This V-TM can be used for TBMs, as it is highly adaptable in diverse geological environments [16, 22]. The proposed V-TM can be reconfigured into an exact-constrained, an over-constrained or an underconstrained configuration according to different configuration modes [16]. For each given configuration, the

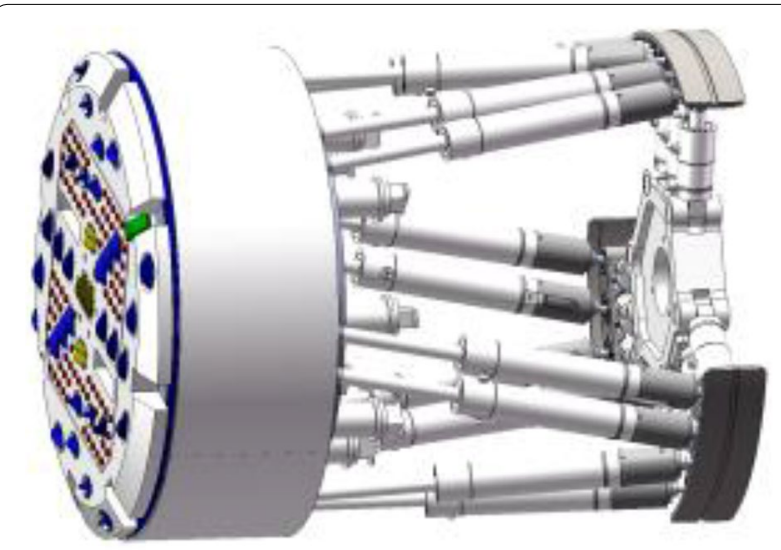

Figure $13 \mathrm{D}$ model of the $\mathrm{V}$-TM system driving mode of each hydraulic cylinder can be reset, depending on the design demand. Thus, millions of thruster configurations can be obtained, and thousands of V-TM working states for each configuration can be set respectively. Based on the proposed method, the structural stiffness of the V-TM can be altered. Nonetheless, the complexity of its configurations and the diversity of its working states lead to difficulty for the structural stiffness algorithm, resulting in further challenges for calculating the payload compliance index and the environment adaptability index. To solve this problem efficiently, an effective and convenient stiffness analytical equation needs to be developed.

The TMs are typical parallel equipment, and the calculation and optimization of their performance indexes are related to the velocity Jacobian matrix and the structural stiffness matrix $[4,9,12,23]$. Therefore, the rigidity characteristics become one of the primary characteristics of a parallel kinematic machine (PKM), and so is for the TM. The main purposes of use are as follows: (1) for general purpose [12, 23], (2) analyzing performance [9, 2427], (3) passive compliance or force control [28, 29], (4) rigid body matching [30, 31], and (5) the analysis of TM deformation.

It is important to consider that in establishing the stiffness analytical model of the proposed V-TM, existing approaches are somewhat different from that of the general reconfigurable PKM [32-36]. For redundant П-TM (type- $\Pi$ thruster mechanisms, two adjacent hydraulic cylinders are parallel), one of the modeling methods based on the virtual displacement theorem is proposed by Ref. [4], another modeling method is also offered by Ref. [36]. The former avoids the problem for calculating the TM's reflecting force, while the latter simplifies the TM's structural complexity. For the V-TM, the modeling method based on PKM is also proposed [4]. In this paper, a reconfigurable thruster with a rigid modeling method is discussed and developed.

The remainder of this paper is organized as follows: To solve the problem, the physical model is first established in Section 2.1. The configuration matrix is then used to describe the relationship between propelling jacks being reconfigured. Section 2.2 details the pattern vectors adopted to describe the driving modes of each hydraulic cylinder. Section 3 concentrates on the V-TM rigidity modeling method with the dynamic compatible equation, the kinematic harmonizing equation and the stiffness analytical equation derived in Sections 3.3, 3.4 and 3.5 respectively.

The proposed solution will provide an effective algorithm to support structural stiffness analysis of the V-TM, and lay a solid theoretical foundation for calculating the performance indexes of the V-TM. Based on the 
proposed stiffness analytical equations derived, the stiffness characteristics of typical configurations and different working status are then analyzed in Section 4, with a discussion of the main factors affecting structural stiffness of the V-TM. Finally, we present a summary of our conclusions in Section 5.

\section{Physical Modeling of the V-TM}

This section focuses on the physical modeling method of the V-TM with a description of kinematic parameters and reconfiguration.

\subsection{Kinematic Parameters of the V-TM}

Figure 1 represents the 3D solid model of the V-TM system discussed here. This consists of gripper mechanics, 12 hydraulic cylinders, and a supporting ring. Each hydraulic cylinder is a branch with an SPS kinematic chain, where $\mathrm{S}$ and $\mathrm{P}$ represent a spherical joint and a prism joint respectively. To establish its physical model, two coordinate systems are appointed to the FP (fixed platform, when the cut plate of a TBM is tuning, the grippers of the TBM are fixed on the support surface) and the MP (moving platform, the support ring is advanced by the thruster). The kinematic sketch of the model is shown in Figure 2. The local coordinate system $O-x y z$ attaches to the FP, and the global coordinate system $P-x^{\prime} y^{\prime} z^{\prime}$ attaches to the MP respectively. The letter $\boldsymbol{q}$ represents the joint variable in Eq. (1):

$$
\boldsymbol{q}=\left\{l_{1}, l_{2}, \ldots, l_{n}\right\}^{\mathrm{T}},
$$

where $l_{i}(i=1,2, \ldots, n)$ denotes the length of the $i$ th leg (or the $i$ th jack), i.e., the distance between the center of the two spherical hinges on the $i$ th propelling jack.

As shown in Figure 3, the coordinate of the spherical joint $\boldsymbol{A}_{i}(i=1,2, \ldots, n)$ on the FP of the V-TM is described in the global coordinate system $O-x y z$ (as shown in

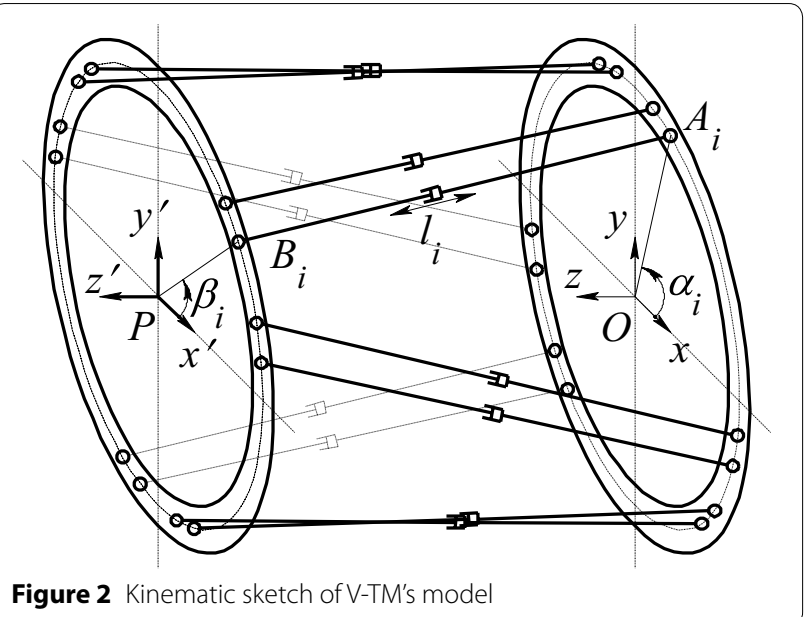

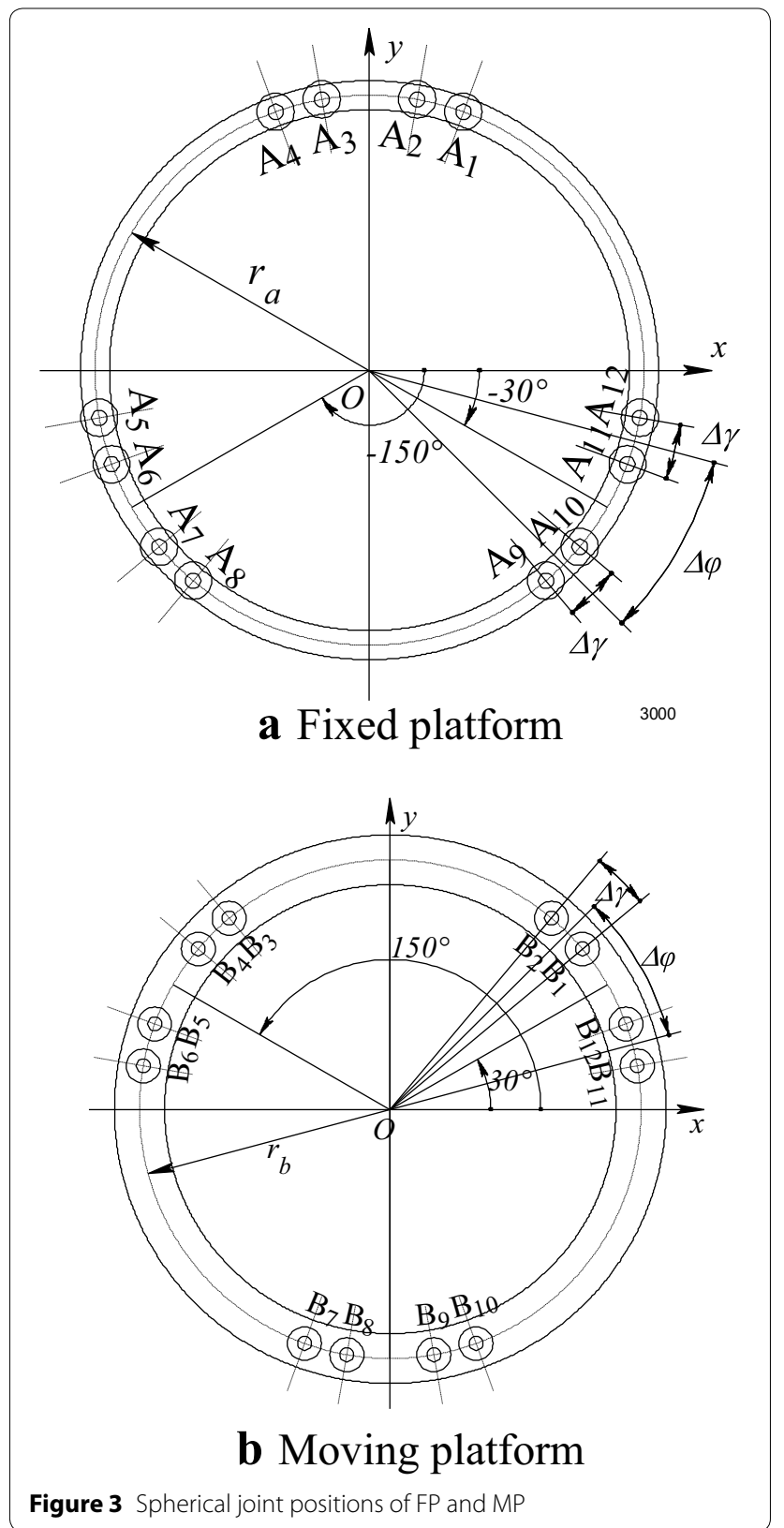

Figure 3a). The coordinate of the spherical joint $\boldsymbol{B}_{i}(i=1$, $2, \ldots, n)$ on the MP of the V-TM is described in the local coordinate system $P-x^{\prime} y^{\prime} z^{\prime}$ (as shown in Figure $3 b$ ). Thus, we obtain:

$$
\boldsymbol{A}_{i}=r_{a}\left\{\begin{array}{l}
\cos \alpha_{i} \\
\sin \alpha_{i} \\
0
\end{array}\right\}, \boldsymbol{B}_{i}=r_{b}\left\{\begin{array}{l}
\cos \beta_{i} \\
\sin \beta_{i} \\
0
\end{array}\right\},
$$

where the direction angles $\alpha_{i}$ and $\beta_{i}$ of the spherical joints' position can be described by two parameters respectively. They represent the intersection angle $\Delta y$ between 
twin legs, and the intersection angle $\Delta \phi$ between two adjacent groups. Let us consider Figure 3 as an example, $\Delta \phi_{a}=30^{\circ}, \Delta \gamma_{a}=10^{\circ}$ in Figure 3a, and $\Delta \phi_{b}=30^{\circ}, \Delta \gamma_{b}=10^{\circ}$ in Figure 3b. Thus, we obtain:

$$
\left\{\begin{aligned}
\alpha_{i}= & 90^{\circ}+120^{\circ}[(i-1) / 4] \\
& +(-1)^{[(i+1) / 2]} \Delta \varphi_{a} / 2+(-1)^{i} \Delta \gamma_{a} / 2, \\
\beta_{i}= & 90^{\circ}+120[(i-1) / 4] \\
& +(-1)^{[(i+1) / 2]}\left(120^{\circ}-\Delta \varphi_{b}\right) / 2+(-1)^{i} \Delta \gamma_{b} / 2,
\end{aligned}\right.
$$

where $i(i=1,2, \ldots, n)$ represents the serial number of the propelling jack. Let

$$
\gamma=\left|\alpha_{i}-\beta_{i}\right|
$$

where $\gamma$ denotes the wedge angle, and represents the differential value between the direction angle of the corresponding spherical joint $\boldsymbol{A}_{i}$ on the FP and the joint $\boldsymbol{B}_{i}$ on the MP, which is the main characteristics parameter of the V-TM.

To describe the location of the MP, we name $X$ as the generalized displacement (sometimes called position or location for short):

$$
\boldsymbol{X}=\left\{\begin{array}{l}
P \\
\theta
\end{array}\right\}, \boldsymbol{P}=\left\{\begin{array}{l}
x_{\mathrm{P}} \\
y_{\mathrm{P}} \\
z_{\mathrm{P}}
\end{array}\right\}, \boldsymbol{\theta}=\left\{\begin{array}{l}
\theta_{x} \\
\theta_{y} \\
\theta_{z}
\end{array}\right\},
$$

where $\boldsymbol{P}$ and $\boldsymbol{\theta}$ denote the position of the center point on the FP, and the relative rotation angle of the MP to the FP respectively.

\subsection{Reconfiguration Method of the V-TM}

The model being studied here is not limited to the fixed symmetric structure as shown above; the drives are divided into several groups $m(m=3,4, \ldots)$. As shown in Figure 4, depending on different application requirements, each hydraulic cylinder can become subordinate to different groups through a reconfigurable hydraulic driving system.

Figure 4 demonstrates that two given hydraulic jacks can be manually cut off by a cut-off value, or automatically cut off by an electromagnetic cartridge value based on different reconfiguration requirements. To describe the reconfiguration relationship between the hydraulic cylinders and the driving groups, the configuration matrix $N$ is created, and its element $n_{i j}$ is defined as follows:

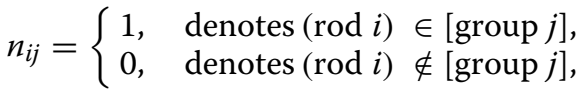

$$
\begin{aligned}
& i=1,2, \ldots, n ; j=1,2, \ldots, m \text {, }
\end{aligned}
$$

where $m$ represents the group number of hydraulic cylinders (same as the number of drivers). Here, several examples of specified configurations are presented in Eq. (7):

$$
\left\{\begin{array}{l}
\boldsymbol{N}_{1}=\left[\boldsymbol{e}_{1}, \boldsymbol{e}_{1}, \boldsymbol{e}_{1}, \boldsymbol{e}_{1}, \boldsymbol{e}_{1}, \boldsymbol{e}_{1}, \boldsymbol{e}_{1}, \boldsymbol{e}_{1}, \boldsymbol{e}_{1}, \boldsymbol{e}_{1}, \boldsymbol{e}_{1}, \boldsymbol{e}_{1}\right] \\
\boldsymbol{N}_{2}=\left[\boldsymbol{e}_{1}, \boldsymbol{e}_{2}, \boldsymbol{e}_{3}, \boldsymbol{e}_{4}, \boldsymbol{e}_{5}, \boldsymbol{e}_{6}, \boldsymbol{e}_{7}, \boldsymbol{e}_{8}, \boldsymbol{e}_{9}, \boldsymbol{e}_{10}, \boldsymbol{e}_{11}, \boldsymbol{e}_{12}\right] \\
\boldsymbol{N}_{3}=\left[\boldsymbol{e}_{1}, \boldsymbol{e}_{1}, \boldsymbol{e}_{2}, \boldsymbol{e}_{2}, \boldsymbol{e}_{3}, \boldsymbol{e}_{3}, \boldsymbol{e}_{4}, \boldsymbol{e}_{4}, \boldsymbol{e}_{5}, \boldsymbol{e}_{5}, \boldsymbol{e}_{6}, \boldsymbol{e}_{6}\right] \\
\boldsymbol{N}_{4}=\left[\boldsymbol{e}_{1}, \boldsymbol{e}_{2}, \boldsymbol{e}_{2}, \boldsymbol{e}_{3}, \boldsymbol{e}_{3}, \boldsymbol{e}_{4}, \boldsymbol{e}_{4}, \boldsymbol{e}_{5}, \boldsymbol{e}_{5}, \boldsymbol{e}_{6}, \boldsymbol{e}_{6}, \boldsymbol{e}_{1}\right] \\
\boldsymbol{N}_{5}=\left[\boldsymbol{e}_{1}, \boldsymbol{e}_{1}, \boldsymbol{e}_{2}, \boldsymbol{e}_{2}, \boldsymbol{e}_{3}, \boldsymbol{e}_{3}, \boldsymbol{e}_{4}, \boldsymbol{e}_{5}, \boldsymbol{e}_{5}, \boldsymbol{e}_{6}, \boldsymbol{e}_{7}, \boldsymbol{e}_{7}\right] \\
\boldsymbol{N}_{6}=\left[0, \boldsymbol{e}_{1}, \boldsymbol{e}_{2}, \boldsymbol{e}_{2}, \boldsymbol{e}_{3}, \boldsymbol{e}_{3}, \boldsymbol{e}_{4}, \boldsymbol{e}_{5}, \boldsymbol{e}_{5}, \boldsymbol{e}_{6}, \boldsymbol{e}_{7}, \boldsymbol{e}_{7}\right]
\end{array}\right.
$$

where $\boldsymbol{e}_{i}$ denotes an $n$-dimension unit vector, $\boldsymbol{N}_{1}$ denotes an under-constrained (or under-driven) configuration, which puts all hydraulic cylinders into one group, has 6 degrees of freedom, but only one driver. $\boldsymbol{N}_{2}$ denotes an over-constrained (over-driven) configuration, which puts each hydraulic cylinder into an individual group, has 6 degrees of freedom, but 12 drivers. $\boldsymbol{N}_{3}$ denotes an exactconstrained configuration, which puts two adjacent and a near hydraulic cylinders into one group and has 6 degrees

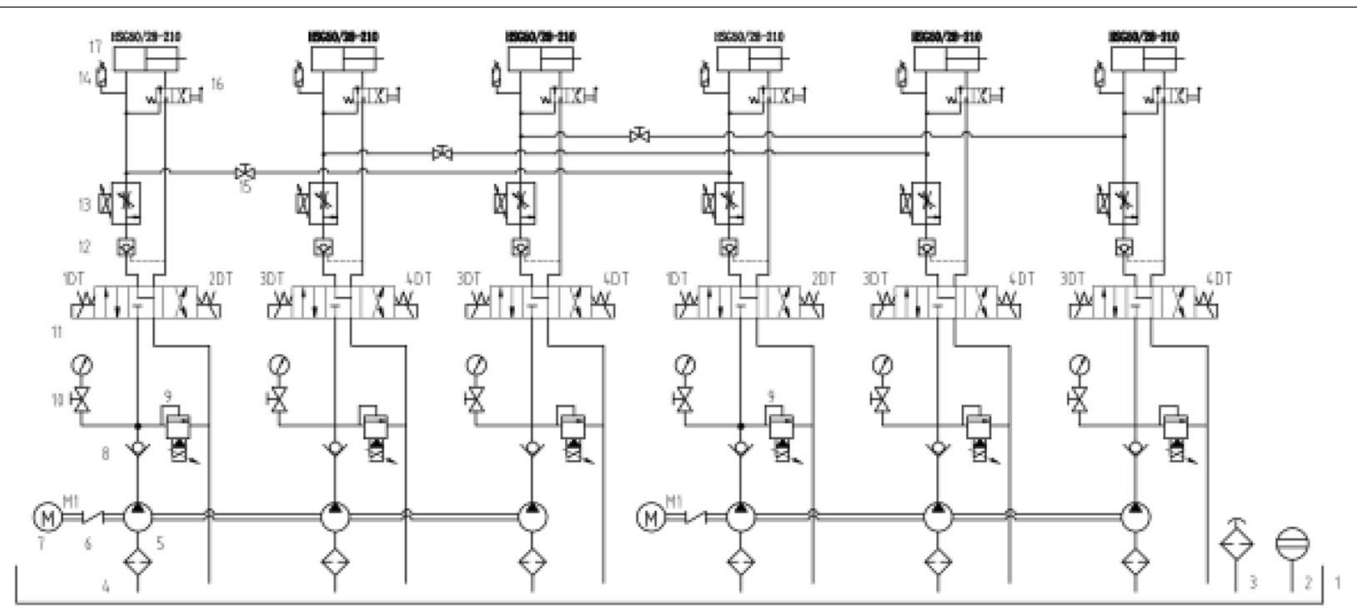

Figure 4 Reconfigurable hydraulic driving system 
of freedom. $\boldsymbol{N}_{4}$ denotes a singularity configuration, which puts two adjacent and afar hydraulic cylinders into one group, has 5 valid degrees of freedom and 1 invalid local degree of freedom, sharing characteristics of both the over-constrained mechanism and the under-constrained mechanism. $\boldsymbol{N}_{5}$ and $\boldsymbol{N}_{6}$ denote two over-constrained configurations with 6 degrees of freedom and 7 drives, except hydraulic cylinder No. 1 , which is not assigned to any group (not in working status) in $\boldsymbol{N}_{6}$.

Besides, two chambers of each hydraulic jack can connect with a two-position three-war value based on different driving modes. Thus, each hydraulic jack could work under both conventional drive mode (or routine drive mode), and differential drive mode. The working pattern vector $\boldsymbol{M}$ describes the driving modes of each hydraulic cylinder. The element $m_{i}$ of vector $\boldsymbol{M}$ is defined as follows:

$$
\begin{aligned}
m_{i} & = \begin{cases}0, & \text { denotes conventional driver mode, } \\
1, & \text { denotes differential driver mode, }\end{cases} \\
i & =1,2, \ldots, n .
\end{aligned}
$$

According to the definitions mentioned above, the mechanism described here consists of $7^{n}$ different configurations in total theoretically, with each configuration possessing $2^{n-k}$ ( $k$ represents the number of hydraulic cylinders not in working states) types of working states. Taking the V-TM described in Figure 3 for instance, there are $7^{12}$ (around $1.38 \times 10^{10}$ ) different configurations in total, assuming all hydraulic cylinders are in working status, there would be $2^{12}(4096)$ different working states in total. Taking the V-TM described in Figure 4 as an example, there are only 3 cut-off valves, thus there are $2^{3}$ different configurations in total, assuming all hydraulic cylinders are in working status, there would be $2^{6}$ different working states in total.

\section{Rigidity Modeling of the V-TM}

This section focuses on the V-TM rigidity modeling method. Based on the physical model of the V-TM established in Section 2, both the dynamic compatible equation between the propeller forces of the hydraulic cylinders and driving forces, and the kinematic harmonizing equation between the hydraulic cylinder displacements and their deformations will be established. The stiffness analytical equation will then be derived using Hooke's law and the Jacobian Matrix.

These equations will provide an effective algorithm to support structural stiffness analysis, and lay a solid theoretical foundation for calculating the performance indexes of the V-TM.

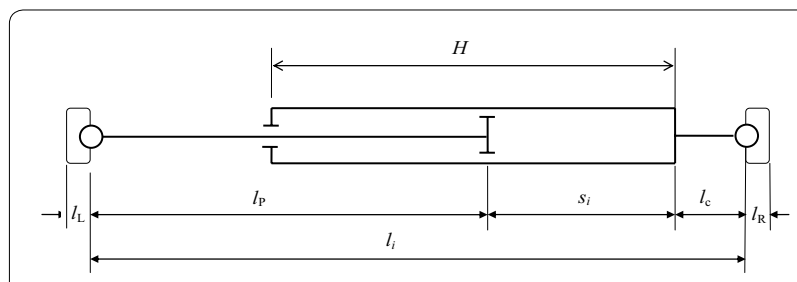

Figure 5 Kinematic sketch of type SPS-Cylinder

\subsection{Rigidity Modeling of the Propelling Jack}

This section will explain the modeling method for each jack in the V-TM, which consists of a type SPS hydraulic cylinder. Its kinematic sketch is described in Figure 5. According to Ref. [4] its stiffness calculation formula is Eq. (9):

$$
\frac{1}{k_{\mathrm{q} i}}=\frac{4\left(s_{i}+l_{\mathrm{e}}\right)}{E_{\mathrm{H}} \pi d_{\mathrm{C}}^{2}}+\frac{4 l_{\mathrm{P}}}{E_{\mathrm{S}} \pi d_{\mathrm{P}}^{2}}+\frac{4 l_{\mathrm{c}}}{E_{\mathrm{S}} \pi d_{\mathrm{c}}^{2}},
$$

where $k_{\mathrm{q} i}, l_{i}$ and $s_{i}(i=1,2, \ldots, n)$ represent the stiffness, length and displacement of the $i$ th $(i=1,2, \ldots, n)$ hydraulic cylinder. $d_{\mathrm{c}}$ represents the internal diameter of the cylinder block, $l_{\mathrm{p}}$ and $d_{\mathrm{p}}$ represent the length and the external diameter of the piston rod. $l_{\mathrm{C}}$ and $d_{\mathrm{C}}$ represent the length and external diameter of the cylinder joint, $E_{\mathrm{H}}$ and $E_{\mathrm{S}}$ represent the elastic modulus of the hydraulic oil and the steel, and $l_{\mathrm{e}}$ represents the equivalent hydraulic cylinder length of the hydraulic circuit, respectively. When hydraulic cylinders are working in differential drive mode, the equation should also consider the factor that the hydraulic oil extrudes from the chamber with the piston rod to the chamber without the piston rod. According to the deformation compatible conditions of the hydraulic oil, we can obtain:

$$
\frac{1}{k_{\mathrm{q} i}}=\frac{4 s_{i} d_{\mathrm{C}}^{2}+4\left(H-s_{i}\right) d_{\mathrm{P}}^{2}}{E_{\mathrm{H}} \pi\left(d_{\mathrm{C}}^{2}-d_{\mathrm{P}}^{2}\right)^{2}}+\frac{4 l_{\mathrm{e}}}{E_{\mathrm{H}} \pi d_{\mathrm{C}}^{2}}+\frac{4 l_{\mathrm{P}}}{E_{\mathrm{C}} \pi d_{\mathrm{P}}^{2}}+\frac{4 l_{\mathrm{C}}}{E_{\mathrm{S}} \pi d_{\mathrm{C}}^{2}},
$$

where $H$ represents the stroke of the hydraulic cylinder. Combining Eq. (10) with the hydraulic cylinder driving mode definition Eq. (8), we obtain:

$$
\begin{aligned}
\frac{1}{k_{q i}}= & \frac{s_{i} \pi d_{\mathrm{C}}^{2}+(H-s)\left(1-m_{i}\right) \pi d_{\mathrm{P}}^{2}}{8 E_{\mathrm{H}}\left(A_{i}^{e}\right)^{2}} \\
& +\frac{4 l_{\mathrm{e}}}{E_{\mathrm{H}} \pi d_{\mathrm{C}}^{2}}+\frac{4 l_{\mathrm{P}}}{E_{\mathrm{C}} \pi d_{\mathrm{P}}^{2}}+\frac{4 l_{\mathrm{C}}}{E_{\mathrm{S}} \pi d_{\mathrm{C}}^{2}},
\end{aligned}
$$

where $A_{i}^{\mathrm{e}}=\pi\left(d_{\mathrm{C}}^{2}-m_{i} d_{\mathrm{P}}^{2}\right)$ represents the effective area of the hydraulic cylinder, Eq. (11) is the stiffness equation for the type SPS hydraulic cylinder. 


\subsection{Kinematics and Dynamics of the V-TM}

As a type of PKM, the inverse kinematic solution of the $\mathrm{V}$-TM, is easily obtained according to the geometric equations.

$$
\left\{\begin{array}{l}
l_{i}=\left\|\boldsymbol{R} \boldsymbol{B}_{i}+\boldsymbol{P}-\boldsymbol{A}_{i}\right\|, \\
\boldsymbol{u}_{i}=\left(\boldsymbol{R} \boldsymbol{B}_{i}+\boldsymbol{P}-\boldsymbol{A}_{i}\right) / l_{i},
\end{array} \quad i=1,2, \ldots, n,\right.
$$

where $\boldsymbol{P}$ and $\boldsymbol{R}$ represent the center position and the orientation matrix of the MP, $\boldsymbol{u}_{i}$ is the direction vector of the $i$ th hydraulic cylinder. According to the differential kinematic principle, we get:

$$
\dot{\boldsymbol{l}}=\boldsymbol{H} \dot{\boldsymbol{X}}, \boldsymbol{H}^{\mathrm{T}}=\left[\begin{array}{ccccc} 
& \boldsymbol{u}_{1} & \boldsymbol{u}_{2} & \cdots & \boldsymbol{u}_{n} \\
\boldsymbol{R} \boldsymbol{B}_{1} \times \boldsymbol{u}_{1} & \boldsymbol{R} \boldsymbol{B}_{2} \times \boldsymbol{u}_{2} & \cdots & \boldsymbol{R} \boldsymbol{B}_{n} \times \boldsymbol{u}_{n}
\end{array}\right]_{6 \times n},
$$

where $\boldsymbol{H}$ represents the velocity inverse Jacobian matrix, each column of $\boldsymbol{H}$ represents the unit linear vector of the $i$ th leg (in Plücker coordinate); this describes the relationship between the joint velocity $\dot{\boldsymbol{l}}_{i}$ and the MP's generalized velocity $\dot{\boldsymbol{X}}$. According to the duality relationship between motion and force, we get the basic dynamics equation as follows:

$$
\boldsymbol{F}=\boldsymbol{H}^{\mathrm{T}} \boldsymbol{\tau},
$$

where $\boldsymbol{F}$ represents the generalized force, and $\boldsymbol{\tau}=\left\{\begin{array}{llll}\tau_{1} & \tau_{2} & \ldots & \tau_{n}\end{array}\right\}^{\mathrm{T}}$ represents the joint force.

\subsection{Dynamic Compatible Equation}

As mentioned above, the V-TM studied here could be an over-constrained or an under-constrained configuration, and its driving force must satisfy the following constraint conditions:

$$
P_{i}=P_{j}, \quad \text { when } \sum_{k=1}^{m} n_{i k} n_{j k} \geq 1, \quad i, j=1,2, \ldots, n,
$$

where $P_{i}$ denotes the pressure of the $i$ th jack. We name $\boldsymbol{P}^{*}=\left\{P_{1}^{*} P_{2}^{*} \ldots P_{m}^{*}\right\}^{\mathrm{T}}$ as the driving pressure in each group, according to the principle that the pressure inside the hydraulic cylinders is equal within the same group, we can obtain:

$$
\boldsymbol{P}=N \boldsymbol{P}^{*}, \quad p_{i}=\tau_{i} / A_{i}^{e},
$$

where $N$ represents the configuration matrix defined in Eq. (6). This equation has described the relationship between the driving forces in each group and the joint force $\tau_{i}$. Therefore, we obtain:

$$
\boldsymbol{\tau}^{*} \cdot \boldsymbol{u}^{*}=\left(\boldsymbol{N}^{\mathrm{T}} \boldsymbol{\tau}\right) \cdot \boldsymbol{u}, \quad \tau_{i}^{*}=A_{i}^{*} P_{i}^{*},
$$

where $\tau_{i}^{*}, A_{i}^{*}$ represent the $i$ th joint force and effective area of the equivalent hydraulic cylinder.

\subsection{Kinematic Harmonizing Equation}

By subset Eq. (17) to Eq. (16), we get:

$$
\boldsymbol{A}^{*} \delta \boldsymbol{l}^{*}=\boldsymbol{N}^{\mathrm{T}} \boldsymbol{A}^{e} \delta \boldsymbol{l},
$$

where $\delta \boldsymbol{l}$ and $\delta \boldsymbol{l}^{*}$ represent the micro or virtual displacement of each hydraulic cylinder and each group. Furthermore, considering the hydraulic oil could flow freely among different hydraulic cylinders within the same group. This will result in:

$$
\boldsymbol{N}^{\mathrm{T}} \delta \boldsymbol{l}^{0}=\{\mathbf{0}\},
$$

where $\delta \boldsymbol{l}^{0}=\left\{\delta l_{1}^{0}, \delta l_{2}^{0}, \ldots, \delta l_{n}^{0}\right\}^{\mathrm{T}}$, and $\delta l_{i}^{0}$ represents the micro displacement equivalent to the flux in and out of the $i$ th hydraulic cylinder. Eq. (18) and Eq. (19) are the kinematic harmonizing equations of the V-TM.

\subsection{Stiffness Equation of the V-TM}

According to Hooke's law for single DOF, we can obtain the basic stiffness equations of the hydraulic cylinders as follows:

$$
\tau_{i}=k_{\mathrm{q} i}\left(\delta l_{i}-\delta l_{i}^{0}\right), \quad \boldsymbol{K}_{\mathrm{q}}=\operatorname{diag}\left\{k_{\mathrm{q} 1}, k_{\mathrm{q} 2}, \ldots, k_{\mathrm{q} n}\right\},
$$

where the differential value between $\delta l_{i}$ and $\delta l_{i}^{0}$ is the real deformation of hydraulic oil in the $i$ th hydraulic cylinder. According to Hooke's law for a mechanism, we can obtain the stiffness equation of the moving platform as follows:

$$
\boldsymbol{F}=\boldsymbol{K} \delta \boldsymbol{X}
$$

where $K$ and $\delta X$ represent the stiffness matrix and the micro deformation of the MP. Assume each hydraulic cylinder is driven independently, i.e., $\delta \boldsymbol{l}^{0}=\{\mathbf{0}\}$, according to Eqs. (13)-(21), we can obtain:

$$
\boldsymbol{F}^{\mathrm{T}} \delta \boldsymbol{X}=\boldsymbol{H}^{\mathrm{T}} \boldsymbol{K}_{\mathrm{q}} \boldsymbol{H} \delta \boldsymbol{X} .
$$

The equation above is valid for $\delta \boldsymbol{X}$ being any generalized virtual displacement vector, thus:

$$
\boldsymbol{K}=\boldsymbol{H}^{\mathrm{T}} \boldsymbol{K}_{\mathrm{q}} \boldsymbol{H} .
$$

The equation above is the stiffness equation of the V-TM when each hydraulic cylinder is driven individually. The equation indicates that when each hydraulic cylinder is driven individually, the mechanism stiffness matrix $K$ is the similitude transformation of hydraulic cylinder stiffness matrix $\boldsymbol{K}_{\mathrm{q}}$, and the similitude transformation matrix is the inverse Jacobian matrix $\boldsymbol{H}$. If we 
assume the hydraulic cylinders are driven by groups, according to Eqs. (13)-(21), we can obtain:

$$
\boldsymbol{N}^{\mathrm{T}} \boldsymbol{K}_{q}^{-1} \boldsymbol{\tau}=\boldsymbol{N}^{\mathrm{T}} \delta \boldsymbol{l}=\boldsymbol{N}^{\mathrm{T}} \boldsymbol{H} \boldsymbol{K}^{-1} \boldsymbol{H}^{\mathrm{T}} \boldsymbol{\tau} .
$$

The equation above is valid for any joint force $\boldsymbol{\tau}$, thus:

$$
\boldsymbol{N}^{\mathrm{T}} \boldsymbol{K}_{\mathrm{q}}^{-1} \boldsymbol{N}=\left(\boldsymbol{H}^{\mathrm{T}} \boldsymbol{N}\right)^{\mathrm{T}} \boldsymbol{K}^{-1}\left(\boldsymbol{H}^{\mathrm{T}} \boldsymbol{N}\right) .
$$

By transferring Eq. (25), we get:

$$
\boldsymbol{K}=\left(\boldsymbol{N}^{\mathrm{T}} \boldsymbol{H}\right)^{\mathrm{T}}\left(\boldsymbol{N}^{\mathrm{T}} \boldsymbol{K}_{\mathrm{q}}^{-1} \boldsymbol{N}\right)^{-1}\left(\boldsymbol{N}^{\mathrm{T}} \boldsymbol{H}\right) .
$$

This is the stiffness equation of the V-TM for general purpose. When driven independently, $N$ will be an identity matrix, and the equation can be simplified to Eq. (23).

\section{Study of the Rigidity Characteristics}

Based on the V-TM's stiffness equation described in Section 3, several case studies will be presented. We therefore consider several typical configurations.

For the convenience of experimental verification of the results, the rigidity characteristics of a scaled V-TM with ratio 1:10 to normal size is studied here. The length of its hydraulic cylinder $l_{i}$ is $540 \mathrm{~mm}$. The designed parameters of its MP and FP are as follows: $r_{\mathrm{a}}=403.278 \mathrm{~mm}$, $r_{\mathrm{b}}=378.948 \mathrm{~mm} ; \gamma=20^{\circ} ; \Delta \phi_{\mathrm{a}}=30^{\circ} 0^{\prime} 2^{\prime \prime}, \Delta \gamma_{\mathrm{a}}=11^{\circ} 37^{\prime} 9^{\prime \prime}$; $\Delta \phi_{\mathrm{b}}=56^{\circ} 59^{\prime} 45^{\prime \prime}, \Delta \gamma_{\mathrm{b}}=11^{\circ} 37^{\prime} 9^{\prime \prime}$.

\subsection{Rigidity Characteristics of Single Jack}

This section focuses on a comparison between the rigidity characteristics of a single jack in two different driving modes. The rigidity characteristics of a single jack can be expressed as its linear structural stiffness. Suppose the equivalent hydraulic cylinder length of the hydraulic pipes $l_{\mathrm{e}}=100 \mathrm{~mm}$, according to its stiffness equation (Eq. 11), the stiffness curves of the SPS propelling jack are shown in Figure 6.

Figure 6 demonstrates that the stiffness of the hydraulic cylinder decreases while its displacement (i.e., the position of the hydraulic cylinder) increases, and the stiffness in differential drive mode is obviously lower than the stiffness in conventional drive mode. Let

$$
\xi_{r}=k_{d} / k_{c}
$$

where $k_{\mathrm{d}}, k_{\mathrm{c}}$ and $\xi_{\mathrm{r}}$ represent the stiffness in differential drive mode, the stiffness in conventional drive mode, and their relative value. Then, relative stiffness $\xi_{\mathrm{r}}$ will vary depending on the equivalent hydraulic cylinder length of the hydraulic pipes $l_{\mathrm{e}}$ and the position of the cylinder as shown in Figure 7.

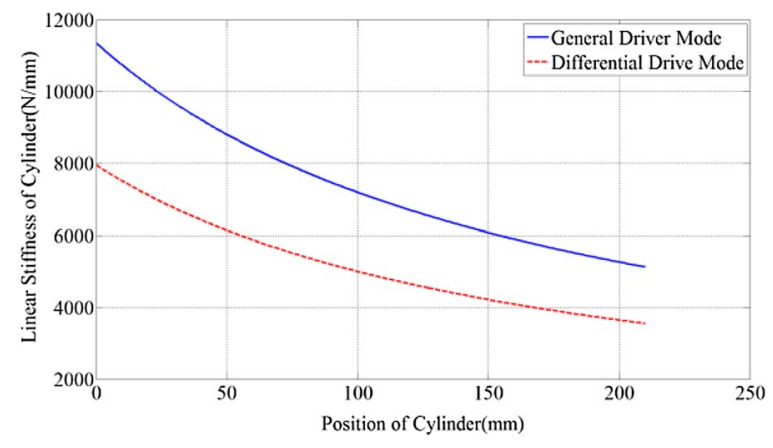

Figure 6 Stiffness curve of SPS propelling jack

Figure 7 demonstrates that given the equivalent hydraulic cylinder length of the hydraulic pipes $l_{\mathrm{e}}$, the position of the cylinder has little effect on the relative stiffness $\xi_{\mathrm{r}}$. Given the position of the cylinder, the relative stiffness $\xi_{\mathrm{r}}$ is proportional to the equivalent hydraulic cylinder length of the hydraulic pipes $l_{\mathrm{e}}$.

\subsection{Rigidity Characteristics of the V-TM's Different Configurations}

This section focuses on the rigidity characteristics and different configurations of the V-TM. For convenience, the three shifting axes are named the $X$-axis, $Y$-axis, and $Z$-axis, and the three rotating axes are named the $A$-axis, $B$-axis, and $C$-axis respectively. By using Eq. (26) in analyzing the typical configuration described in Eq. (7), the V-TM's stiffness matrix can be obtained. The main stiffness of each axis can be used to express its rigidity characteristics, the results are shown in Figure 8.

Figure 8 demonstrates that the stiffness on the $Z$-axis, $A$-axis and $B$-axis is much higher than the stiffness on the $X$-axis, $Y$-axis and $C$-axis. For two anear hydraulic cylinders, the over-constrained drive mode provides them with slightly higher stiffness than the exact-constrained drive mode. For the under-constrained configuration $\boldsymbol{N}_{1}$, its main stiffness is none, zero on the $Z$-axis alone. For the singularity configuration $\boldsymbol{N}_{4}$, its stiffness on the $\mathrm{C}$-axis is zero.

Once the jacks are given and their working states are set, the stiffness along the $Z$-axis is not affected by the reconfiguration or the grouping of hydraulic cylinders. The stiffness along the $Z$-axis could only be altered by deactivating certain propelling jacks (e.g., the configuration $\boldsymbol{N}_{5}$ ) or the working states of the jacks.

\subsection{Rigidity Characteristics of Different Working States of the V-TM's}

This section focuses on the rigidity characteristics of the V-TM's that have worked in different states for the 


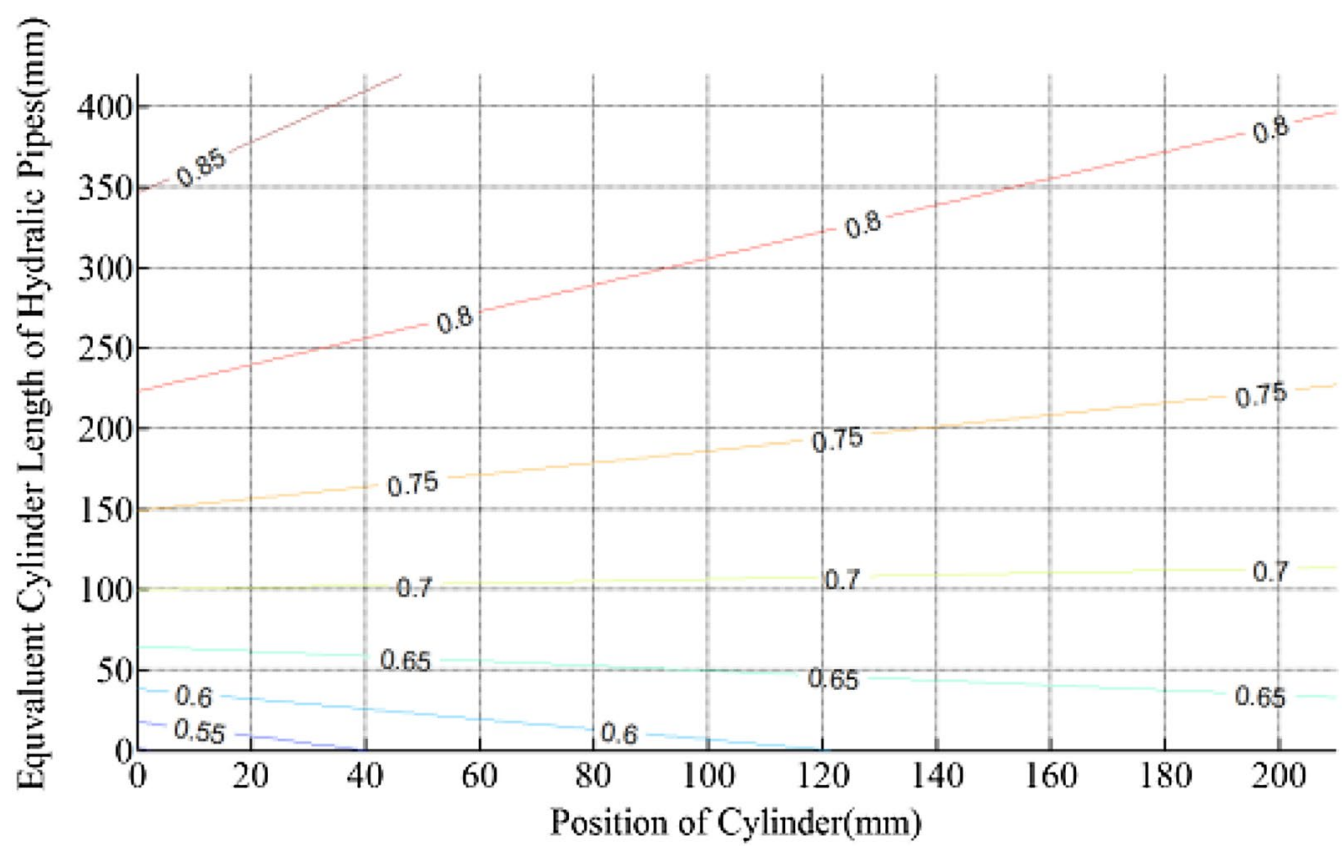

Figure 7 Relative stiffness of SPS propelling jack

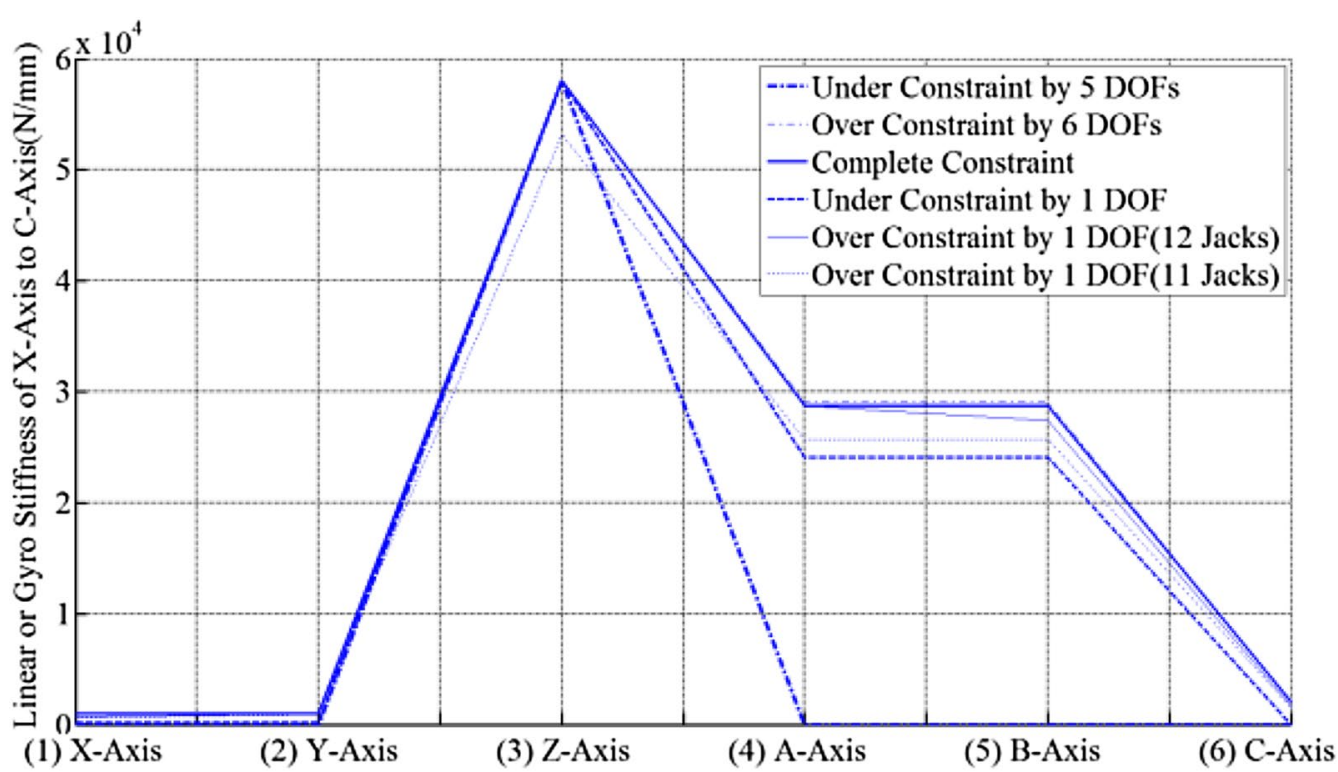

Figure 8 Thrust mechanism stiffness curves of different configurations

given configurations. Two typical configurations will be discussed here. The first is an exact-constrained symmetrical configuration $N_{3}$, and the second is an overconstrained unsymmetrical configuration $N_{6}$. The main stiffness of each axis is used to express its rigidity characteristics as described in Section 4.2.

\subsubsection{Exact-constrained Symmetrical Configuration}

For the configuration $\boldsymbol{N}_{3}$, all propelling jacks participate in propelling duty, and there are $2^{12}=4096$ working states in total. The main stiffness that covers the $X$-axis, $Y$-axis, and $Z$-axis in different working states is shown in Figures 9, 10, 11. 


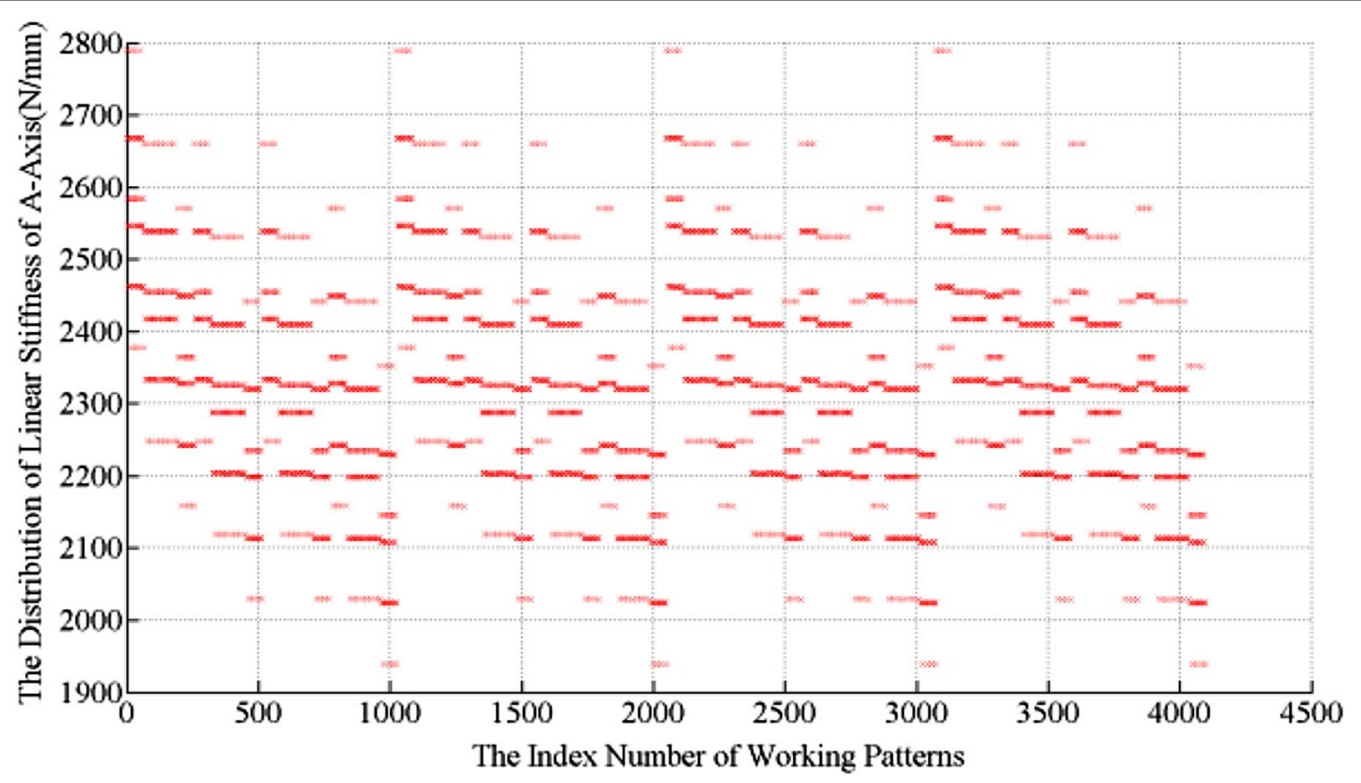

Figure 9 Main stiffness distribution on $X$-axis

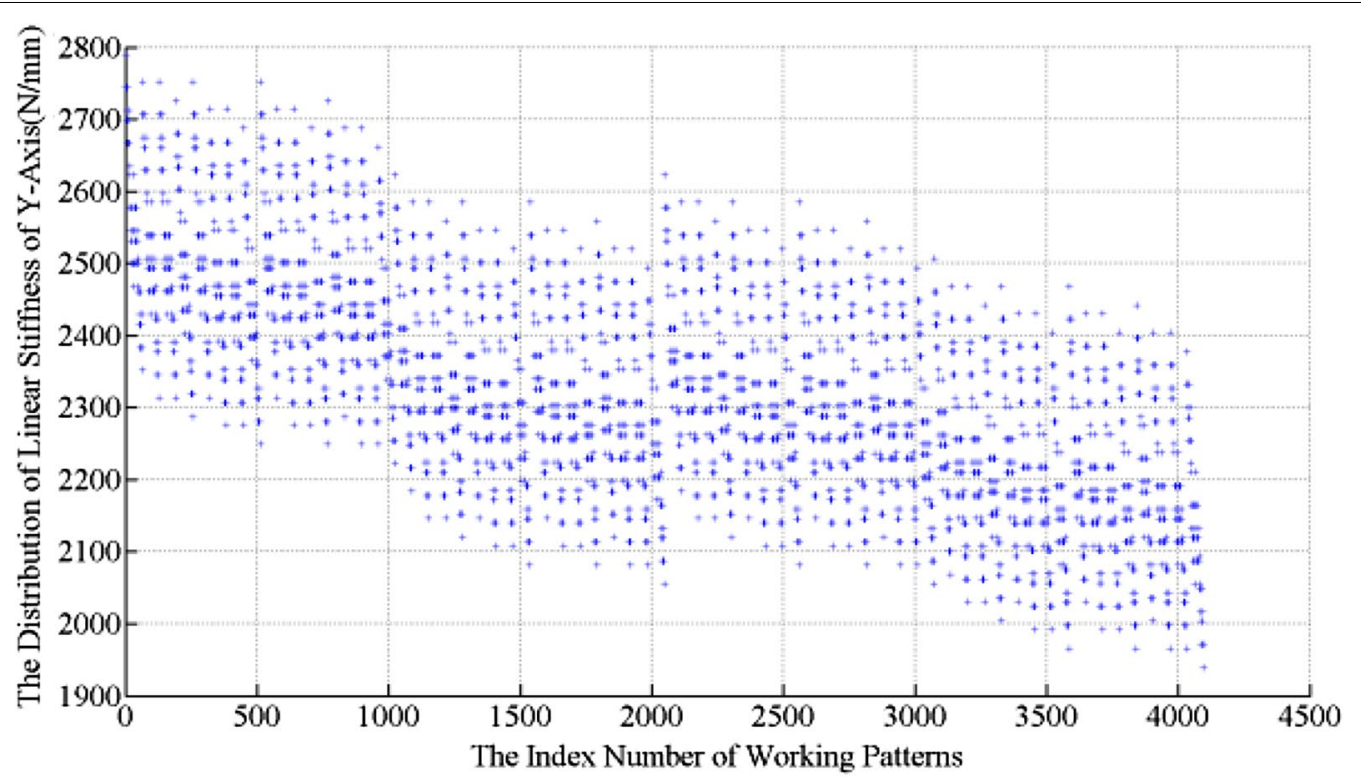

Figure 10 Main stiffness distribution on $Y$-axis

Figures 9, 10, 11 demonstrate that the stiffness on the $Z$-axis is much higher than the stiffness on the $X$-axis and $Y$-axis. The main stiffness on each axis varies substantially via different working states, thus providing an advantage in matching its stiffness. For the configuration described above, the stiffness on the $A$ - axis, $B$-axis and $C$-axis will satisfy:

$$
\xi_{k}=k_{A} / k_{Y}=k_{B} / k_{X}=k_{Z} / k_{C},
$$

where the ratio $\xi_{k}$ is proportional to the position of the $\mathrm{MP}$, and is independent of working states. Let us take configuration $N_{3}$ as an example; the relative stiffness ratio $\left(\xi_{k}\right)$ is defined as the ration of linear stiffness (stiffness of $Z$-axis) to its gyro stiffness (stiffness of $C$-axis). Figure 12 shows that its relative stiffness ratio $\left(\xi_{k}\right)$ is directly proportional to the position of MP (i.e., the length of the hydraulic cylinder). 


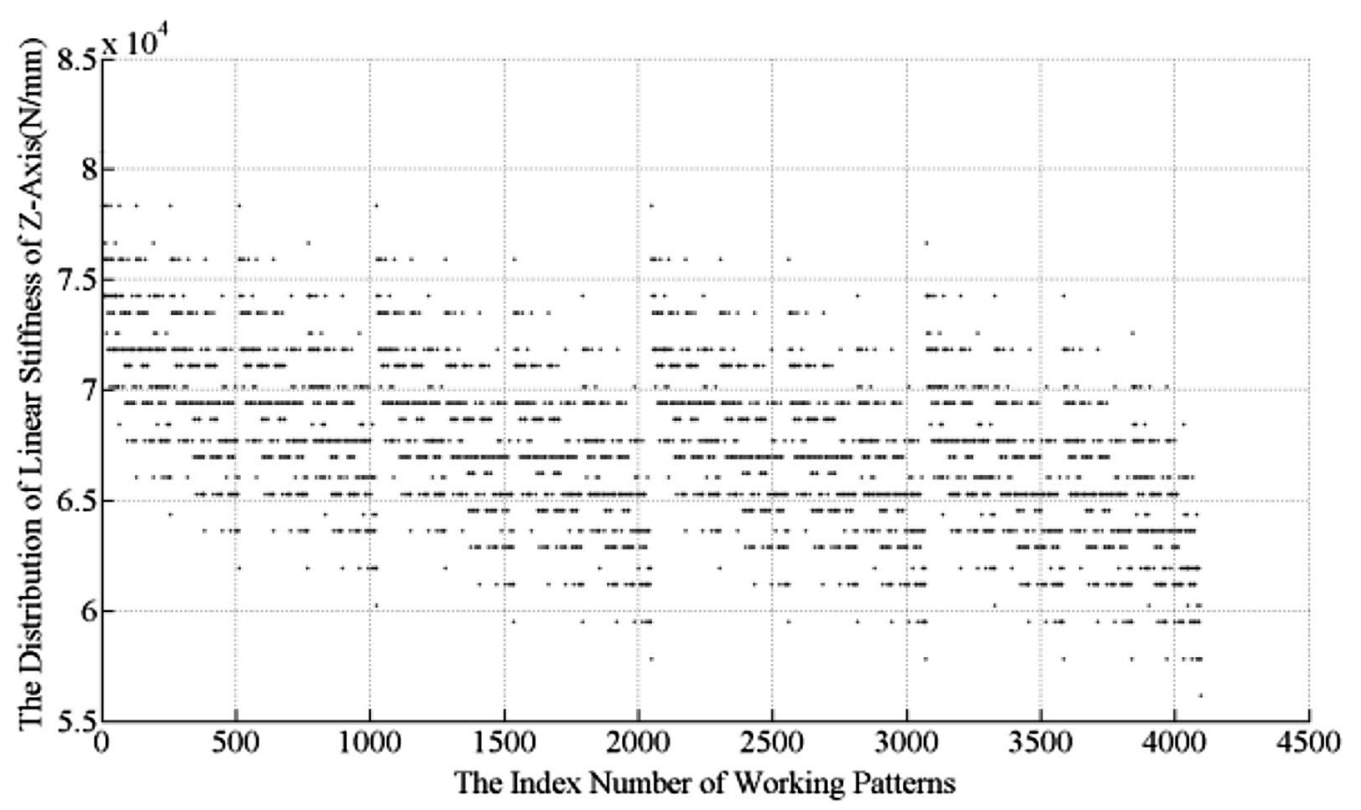

Figure 11 Main stiffness distribution on Z-axis

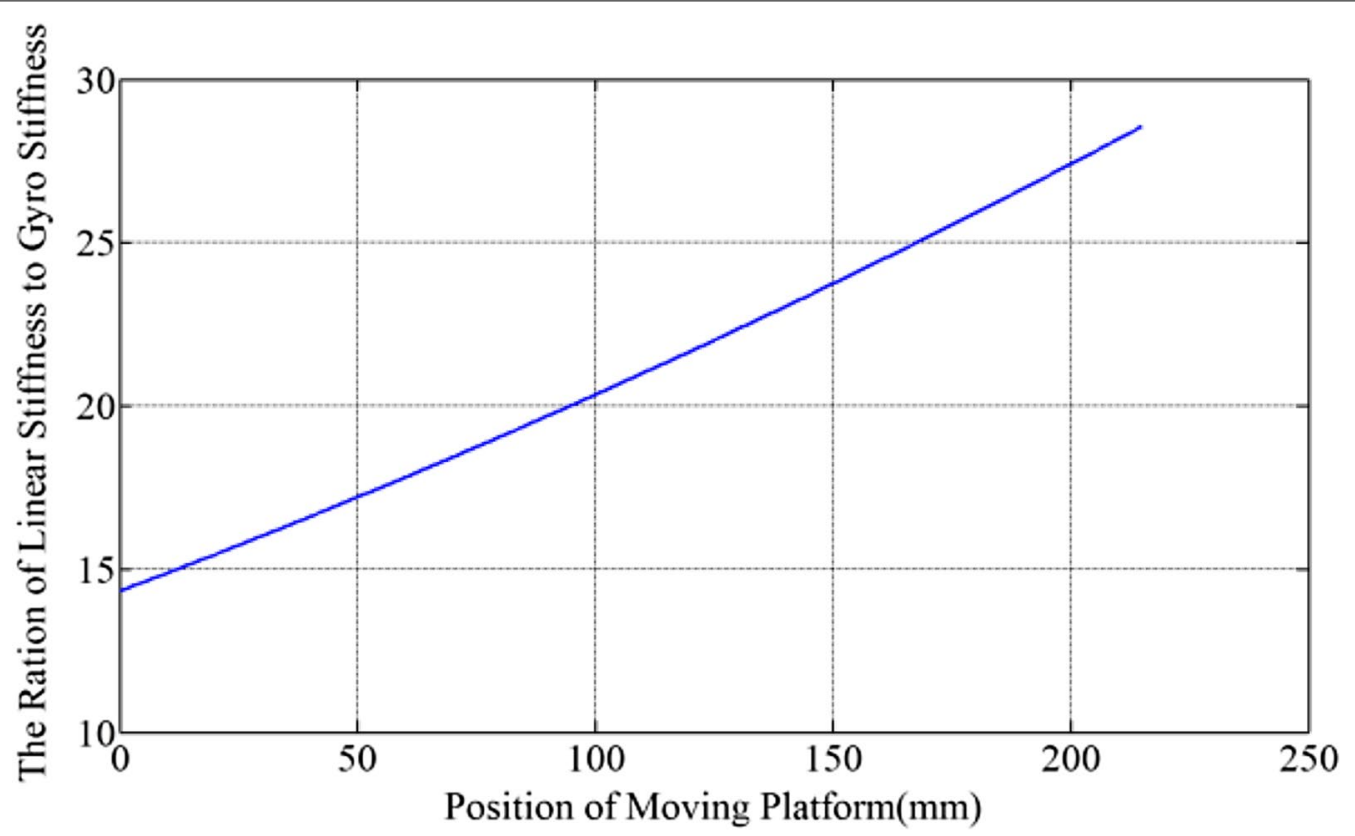

Figure 12 Relative stiffness ratio $\left(\xi_{k}\right)$ of the $N_{3}$ configuration

\subsubsection{Over-constrained Unsymmetrical Configuration}

As mentioned in Section 2.2, the configuration $\boldsymbol{N}_{6}$ described in Eq. (7) has seven drives creating one overconstraint. This configuration has $2^{11}=2048$ working states in total. Its relative stiffness $\xi_{k}$ corresponds to the working states as described in Section 4.3.1, and is shown in Figure 13.

Figure 13 demonstrates that the relative stiffness ratio $\xi_{k}$ is no longer a fixed value, as its distribution varies within a certain range. For the given configuration $N_{6}$, the relative stiffness along the $B$-axis to the $Y$-axis is the 


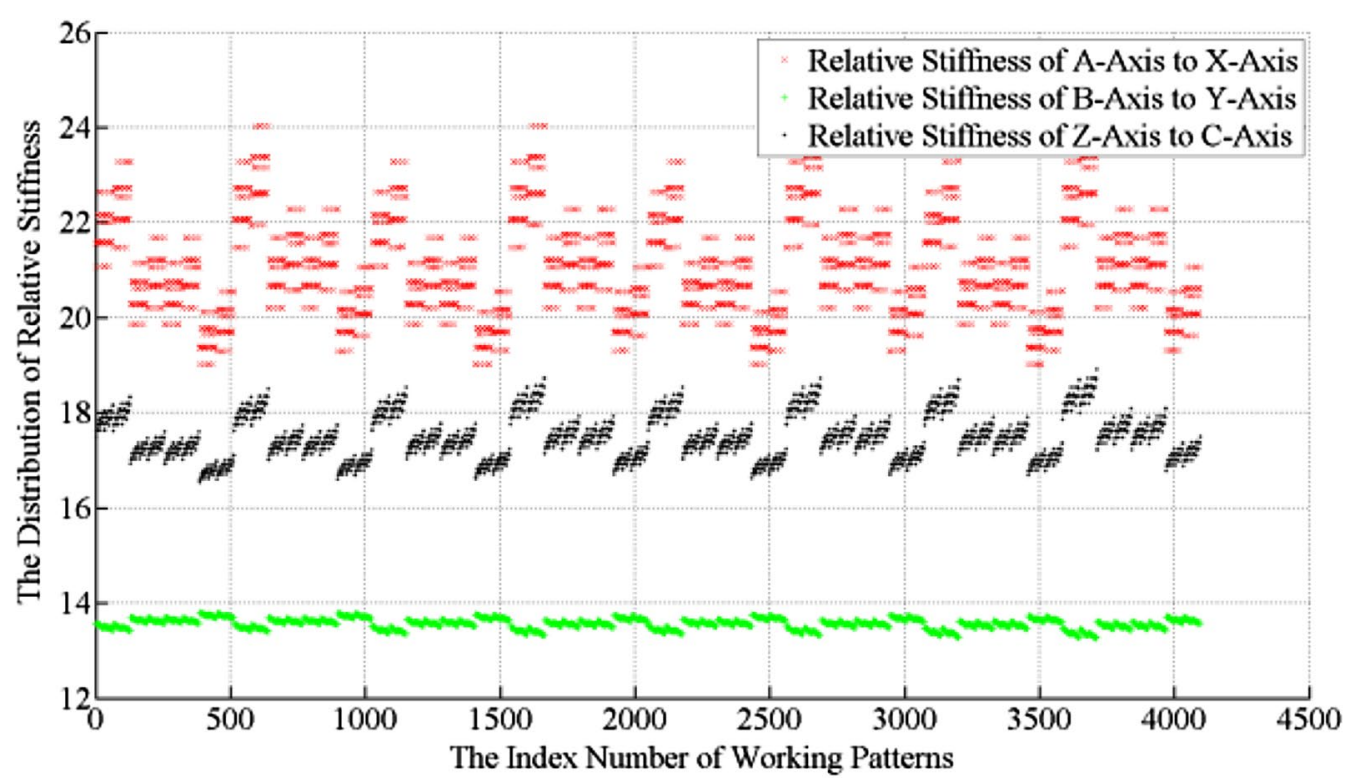

Figure 13 Relative stiffness ratio $\left(\xi_{k}\right)$ of the $\boldsymbol{N}_{6}$ configuration

smallest, with a lower fluctuating range, and the relative stiffness along the $A$-axis to the $X$-axis is the largest, with a higher fluctuating range. The stiffness along the $Y$-axis is obviously smaller than the stiffness along the $X$-axis. This type of configuration is suitable for the uneven turning face of the TBM.

\section{Conclusions}

(1) This research proposes a feasible reconfigurable method with the configuration matrix used to describe the relationship between propelling jacks undergoing reconfiguration. Based upon this, the V-TM can be reconfigured to exact-constrained, over-constrained, and/or under-constrained configurations, and its stiffness can be altered on demand. Furthermore, a feasible method to reset the working states of the hydraulic cylinders of the V-TM has been proposed, with the working pattern vector adopted to describe the driving modes of each hydraulic cylinder. The V-TM can operate in thousands of states, and its stiffness can be adapted to match the requirements of the environment.

(2) Based on the dynamic compatible equation and the kinematic harmonizing equation, the rigidity modeling method of the reconfigurable V-TM is presented, and its concise stiffness equation established. The proposed method provides an effective algorithm for the structural stiffness of the V-TM, and this lays a solid theoretical foundation for calculating performance indexes.
(3) The stiffness of the jack is inversely proportional to its displacement, and the stiffness in differential drive mode is obviously lower than that of the generalized drive mode. This is one of the main factors affecting structural rigidity of the V-TM. When the configuration of the V-TM is provided, via altering the driving modes of each jack, the stiffness along each axis could be efficiently altered, except for the stiffness alone the $Z$-axis.

(4) For two nearby and a near hydraulic cylinders, over-constrained drive mode (in different groups) provides them with slightly higher stiffness than exact-constrained drive mode (in the same group), for their location, deviations are small. However, for two nearby and afar hydraulic cylinders, over-constrained drive mode (in different groups) provides them with obviously lower stiffness than the exactconstrained drive mode (in the same group), for their location, deviations are high. This is another main factor that affects the structural rigidity of the V-TM, and provides useful information for matching and optimizing V-TM structural rigidity.

\section{Authors' Contributions}

$\mathrm{XL}, \mathrm{YX}$ were in charge of the whole trial, and wrote the manuscript; JX assisted with sampling and laboratory analyses. All authors read and approved the final manuscript.

\section{Authors' Information}

Younan Xu, born in 1965, is currently a professor in East China Jiaotong University, China. He received his PhD degree from Tsinghua University, China, in 2013. His research interests include mechanology and robotics. 
Xinjun Liu, Humboldt Research Fellow, Scholars of Yangtze River, born in 1973, is currently a professor and doctoral supervisor in Tsinghua University, China. He received his PhD degree from Yansan University, China, in 1999, and became a post-doctoral researcher in Tsinghua University, China, between 2000 and 2001. His research interests include mechanology, robotics, and advanced manufacturing technology.

Jiyu Xu, born in 1992, is currently a master degree candidate in Department of Mechanical Engineering and Aerospace, Polytechnic of Torin, Italy.

\section{Competing Interests}

The authors declare that they have no competing interests.

\section{Funding}

Supported by National Natural Science Foundation of China (Grant No. 51675180), and National Key Basic Research Program of China (973 Program, Grant No. 2013CB037503).

\section{Author Details}

${ }^{1}$ School of Mechantronics and vehicle Engineering, East China Jiaotong University, Nanchang 330013, China. ${ }^{2}$ Department of Mechanical Engineering, Tsinghua University, Beijing 100084, China. ${ }^{3}$ Department of Mechanical Engineering and Aerospace, Polytechnic of Torin, Torin 10135, Italy.

Received: 20 January 2019 Accepted: 15 November 2019

Published online: 12 December 2019

\section{References}

[1] J Z Huo, W Z Wang, W Sun, et al. The multistage rock fragmentation load prediction model of tunnel boring machine cutter group based on dense core theory. The International Journal of Advanced Manufacturing Technology, 2017, 90(1-4): 277-289.

[2] D Q Hou, G F Gong, H Shi, et al. Compliance characteristics of propulsion system of shield tunneling machine under sudden load. Journal of Zhejiang University (Engineering Science), 2013, 47(3): 522-527. (in Chinese)

[3] J Huo, X Sun, G Li, et al. Multi-degree-of-freedom coupling dynamic characteristic of TBM disc cutter under shock excitation. Journal of Central South University, 2015, 22: 3326-3337.

[4] YN Xu. Research on payload compliance design theory and method for thruster mechanisms of shield machines. Beijing: Tsinghua University, 2012. (in Chinese)

[5] J Z Huo, N Hou, W Sun, et al. Analysis of dynamic characteristics and structural optimization of tunnel boring machine cutter system with multi-joint surface. Nonlinear Dynamics, 2017, 87(1): 237-254.

[6] J Z Huo, W Sun, XY Ouyang, et al. Coupling layout design of disc cutters group and cutterhead supporting structure. Journal of Mechanical Engineering, 2014, 50(21): 23-30. (in Chinese)

[7] HY Sun, W Guo, J Q Liu, et al. Layout design for disc cutters based on analysis of TBM cutter-head structure. Journal of Center South University, 2018, 25: 812-830.

[8] Q Geng, ZY Wei, J Du, et al. Cutters' layout optimization method of the full-face rock tunnel boring machine based on grey relational analysis. Journal of Mechanical Engineering, 2014, 50(21): 45-53. (in Chinese)

[9] Y N Xu, X J Liu, J S Wang. Payload compliance and its design theory for thruster mechanism. Journal of Mechanical Engineering, 2014, 50(21): 60-68. (in Chinese)

[10] D Q Hou, G F Gong, H Shi, et al. Design of new propulsion system of shield tunneling machine based on compliance characteristics. Journal of Zhejiang University (Engineering Science), 2013, 47(7): 1287-1292. (in Chinese)

[11] H Shi, HY Yang, G F Gong, et al. Definition and evaluation method for compliance of thrust hydraulic system for shield tunneling machine. Journal of Zhejiang University (Engineering Science), 2013, 47(8): 1444-1449. (in Chinese)

[12] R A Snowdon, M D Ryley, J Temporal. The effect of hydraulic stiffness on tunnel boring machine performance. Int. J. Rock Mech. Min. Sci. \& Geomech. Abstra., 1983, 20(5): 203-214.
[13] Y L Cheng, J Zhong, ZY Ji, et al. Geological adaptive design method and application of TBM cutterhead. Journal of Mechanical Engineering, 2018, 54(1): 1-9. (in Chinese)

[14] C J Shao, J F Liao, Z T Liu, et al. Trajectory tracking control in horizontal direction of open-type hard rock tunnel boring machine based on adaptive robust control strategy. Journal of Mechanical Engineering, 2018, 54(1): 113-119. (in Chinese)

[15] M Yue, L Guo. Double closed-loop adaptive rectification control of a shield tunneling machine with hydraulic actuator dynamics subject to saturation constraint. Journal of Vibration \& Control, 2014, 22(2): 608-614.

[16] Y N Xu, X J Liu, J Y Xu. A kind of reconfigurable thrusting-grippingregripping TBM device with variable stiffness and 1 to 6 DOFs: CN, ZL 201720855999.7. 2018-05-11[2019-10-08]. http://cprs.patentstar.com. $\mathrm{cn} /$ Search/Detail?ANE =AHIA6FBA7FBA9ACA9GDC6FBAHIIA9EGG9DC B9GAG9ECGBBIA. (in Chinese)

[17] K S Deng, X Q Tang, L P Wang, et al. Optimization of thrust system cylinder layout for shield machine for random load. Journal of Tsinghua University (Science and Technology), 2010, 50(8): 1248-1252.

[18] Y Zhao, H Wang, H D Yu, et al. Evaluation method for mechanical system of a shield TBM based on stiffness matching. Science China: Science and Technology, 2012, 42(10): 1228-1237. (in Chinese)

[19] K S Deng, Y Y Li, Z R Yin. Thrust distribution characteristics of thrust systems of shield machines based on spatial force ellipse model in mixed ground. Journal of Mechanical Science and Technology, 2016, 30 (1): 279-286.

[20] K S Deng, Z R Yin, B L Meng. A load-balancing-oriented symmetrical uneven layout design for thrust system in tunneling machines under composite ground. Journal of Mechanical Engineering Science, 2019, 233(8): 8477-2854.

[21] K S Deng, L Zhang, Y C Ding, et al. Layout optimization for non-equidistant thrust system of tunneling machine based on geometric progression under mixed ground. Science Progress, 2019: 1-17.

[22] Y N Xu, X J Liu, JY Xu. A kind of fully automatic thrusting-gripping-regripping TBM device with bi-hexapod thrusters and bi-tripod grippers: CN, ZL201710575228.7.2019-05-03 [2019-10-08]. http://cprs.patentstar.com. $\mathrm{cn} /$ Search/Detail?ANE $=6$ EAA9CIB6BEA9BFE9FFC9GDA9AEA9BEDBFI AAHIA8CEA9FCG. (in Chinese)

[23] J Temporal, R A Snowdon. The effect of hydraulic stiffness on tunnel machine performance. Tunnels and Tunneling, 1982, 14(2): 11-13.

[24] W R Southern, C G Lyons. The study of a passive accommodation device in robotic insertion processes. Journal of Materials Processing Technology, 2002, 124: 261-266

[25] MXWang, HT Liu, T Huang, et al. Compliance analysis of a 3-SPR parallel mechanism with consideration of gravity. Mechanism and Machine Theory, 2015, 84, 99-112.

[26] K S Deng, B L Meng, C Xiang. Adaptability to stratum characteristics for layout of thrust system in tunneling machines based on variation coefficient. Advances in Mechanical Engineering, 2016, 8(12): 1-9.

[27] K S Deng, C Xiang, B L Meng, et al. A force transmission assessment method for thruster system in shield machines based on the relative coefficient in compound ground. Automation in Construction, 2017, 83: 353-359.

[28] H S Kim, J J Park, J B Song, et al. Design of safety mechanism for an industrial manipulator based on passive compliance. Journal of Mechanical Science and Technology, 2010, 24 (11): 2307-2313.

[29] K S Deng, X M Zhang, J S Yang, et al. Deformation characteristics under variable stiffness for propelling mechanism of EPB shield machines in mixed ground. Journal of Mechanical Science and Technology, 2014, 28(9): 3679-3685

[30] J K Salisbury, J Craig. Articulated hands: Kinematic and force control issues. International Journal of Robotica Research, 1982, 1(1): 4-17.

[31] D E Whitney. Quasi-static assembly of compliantly supported rigid parts. ASME J. Dyn. Syst. Measurement Control, 1982, 104(1): 65-77.

[32] WYe, Y F Fang, S Guo. Design and analysis of a reconfigurable parallel mechanism for multidirectional additive manufacturing. Mechanism and Machine Theory, 2017, 112: 307-326.

[33] D M Gan, J Dias, L Seneviratne. Unified kinematics and optimal design of a 3rRPS metamorphic parallel mechanism with a reconfigurable revolute joint. Mechanism and Machine Theory, 2016: 96-111. 
[34] B Wang, Y F Fang. Geometric constraint and mobility analysis of a reconfigurable parallel mechanism. Journal of Mechanical Engineering, 2018, 54(7): 28-37. (in Chinese)

[35] W Ye, Y F Fang, S Guo, et al. Design of reconfigurable parallel mechanisms with discontinuously movable mechanism. Journal of Mechanical Engineering, 2015, 51(13): 137-143. (in Chinese)
[36] Y C Deng, W Z Guo, F Gao. Equivalent mechanism-based modeling for grouping performance analysis of the thrust system of shield machines. Journal of Mechanical Engineering, 2010, 46(13): 123-127. (in Chinese)

\section{Submit your manuscript to a SpringerOpen ${ }^{\circ}$ journal and benefit from:}

- Convenient online submission

- Rigorous peer review

- Open access: articles freely available online

- High visibility within the field

- Retaining the copyright to your article

Submit your next manuscript at $\boldsymbol{\sim}$ springeropen.com 